\title{
BMJ Open How is cervical cancer screening information communicated in UK websites? Cross-sectional analysis of content and quantitative presentation formats
}

\author{
Yasmina Okan (1) , ${ }^{1}$ Samuel G Smith, ${ }^{2}$ Wändi Bruine de Bruin (1D ${ }^{1,3}$
}

To cite: Okan Y, Smith SG, Bruine de Bruin W. How is cervical cancer screening information communicated in UK websites? Crosssectional analysis of content and quantitative presentation formats. BMJ Open 2019;9:e029551. doi:10.1136/ bmjopen-2019-029551

- Prepublication history and additional material for this paper are available online. To view these files, please visit the journal online (http://dx.doi. org/10.1136/bmjopen-2019029551).

Received 30 January 2019 Revised 03 September 2019 Accepted 13 September 2019

Check for updates

(C) Author(s) (or their employer(s)) 2019. Re-use permitted under CC BY. Published by BMJ.

${ }^{1}$ Centre for Decision Research, Leeds University Business School, University of Leeds, Leeds, UK

${ }^{2}$ Leeds Institute of Health Sciences, University of Leeds, Leeds, UK

${ }^{3}$ Department of Engineering and Public Policy, Carnegie Mellon University, Pittsburgh, Pennsylvania, USA

Correspondence to Dr Yasmina Okan; y.okan@leeds.ac.uk

\section{ABSTRACT}

Objectives To investigate whether UK websites about cervical cancer screening targeted to the public include (1) information about benefits and risks of screening, possible screening results and cervical cancer statistics, (2) quantitative presentation formats recommended in the risk communication literature and (3) appeals for participation and/or informed decision-making.

Design Cross-sectional analysis of websites using a comprehensive checklist of information items on screening benefits, risks, possible results and cervical cancer statistics.

Outcome measures We recorded the number of websites that contained each of the information items, and the presentation format used for probabilistic information (no quantification provided, verbal quantifiers only, different types of numerical formats and/or graphs). We also recorded the number of websites containing appeals for participation and/or informed decision-making.

Setting Websites were identified through the most common Google search terms used in the UK to find information on cervical screening, according to GoogleTrends and a commercial internet-monitoring programme. Two additional websites were identified by the authors as relevant.

Results After applying exclusion criteria, 14 websites were evaluated, including websites of public and private health service providers, charities, a medical society and a pharmacy. The websites mentioned different benefits, risks of screening and possible results. However, specific content varied between websites. Probabilistic information was often presented using non-recommended formats, including relative risk reductions to express screening benefits, and verbal quantifiers without numbers to express risks. Appeals for participation were present in most websites, with almost half also mentioning informed decision-making.

Conclusions UK websites about cervical cancer screening were generally balanced. However, benefits and risks were presented using different formats, potentially hindering comparisons. Additionally, recommendations from the literature to facilitate understanding of quantitative information and facilitate informed decisions were often not followed. Designing websites that adhere to existing recommendations may support informed screening uptake.
Strengths and limitations of this study

- We analysed the content of UK websites about cervical cancer screening using an established checklist of information items, and identified additional information that was mentioned commonly.

- We systematically examined whether cancer screening websites present probabilistic information in formats recommended in the risk communication literature.

- We identified websites by applying the most common Google search terms used in the UK to find information on cervical screening, and examined the majority of links that users will realistically access.

- All the information items we assessed may not be relevant for screening decisions, whereas we may have omitted some information that can be relevant (eg, about the human papillomavirus).

- We did not examine whether websites mentioned the uncertainty associated with estimates of benefits and risks, although such information can be important for informed decision-making.

\section{INTRODUCTION}

Cervical cancer is highly preventable. It is caused in most cases by an infection with the human papillomavirus (HPV), which may lead to abnormal changes in cervical cells. ${ }^{12}$ Such abnormalities can be detected through screening and treated before they become cancerous. ${ }^{3}$ Indeed, cervical screening significantly reduces both cervical cancer incidence and cancer-specific mortality. ${ }^{3-7}$ In the UK, age-standardised incidence was 11.8 in 100 000 in 2014, and age-standardised mortality was 3.3 in $100000 .^{8}$ In England alone, estimates suggest that there would be 1827 additional cervical cancer deaths per year without screening. ${ }^{7}$

However, cervical screening is associated with potential risks, including the detection of indolent abnormal cells that would have 
cleared up on their own (ie, overdiagnosis), potentially leading to unnecessary treatment. ${ }^{9} 10$ A recent meta-analysis indicates that about half of untreated moderately abnormal cells regress within 2 years, ${ }^{9}$ suggesting that overtreatment is relatively common. The removal of large amounts of tissue during treatment can also increase the risk of preterm birth in future pregnancies. ${ }^{11-13}$ However, experts and policy-makers generally agree that benefits of cervical screening outweigh potential risks. ${ }^{51415}$ In the UK, the National Health Service (NHS) offers screening to all women aged 25-64. It has been emphasised that screening invitees need information about both benefits and risks to make informed decisions about participation..$^{14} 1617$

Organised screening programmes often use written outreach materials. In the UK, eligible women receive an invitation letter with an information leaflet that mentions websites about cervical screening. Hence, it is important to examine whether widely accessed websites effectively support decisions about screening participation. Both European and UK guidelines have emphasised that cancer screening communications should be comprehensive in content, and should provide balanced discussions of benefits and risks to screening. ${ }^{17-20}$ Such guidelines have also highlighted that screening communications should be comprehensible, and avoid quantitative presentations that are hard to understand. ${ }^{17-19}$ Communications that are not well understood can cause undue concern, reduce recipients' self-efficacy beliefs about their capacity to participate in screening and undermine informed decision-making. ${ }^{21} 22$

Quantitative information in screening communications, however, can be challenging even for educated audiences. ${ }^{21}{ }^{23}$ Such information can be presented using a range of formats, including verbal quantifiers (eg, 'low' or 'moderate' risk), various numerical formats and graphical displays (eg, icon arrays). Research in risk communication has shown that quantitative presentation formats vary considerably in their effectiveness to promote accurate understanding. Some formats are known to be relatively ineffective, while others still lack conclusive evidence. ${ }^{24}$ Table 1 provides an overview of quantitative formats, associated evidence-based recommendations and supporting references. Key recommendations relevant to screening communications include avoiding the use of verbal quantifiers without accompanying numbers, numerical ' 1 -in- $X$ ' formats and presentations of risk reductions in relative terms. Another recommendation is to add simple graphical displays to numerical information.

Existing guidelines also state that screening communications should not persuade people to attend or present screening as necessary or important, without acknowledging that not participating is a reasonable choice. ${ }^{17-20}$ Recent guidance from the UK National Screening Committee ${ }^{17}$ notes that information should make it clear that it is a personal choice to accept or decline screening and both choices will be fully supported' (p6). This approach to screening communications seeks to respect

Table 1 Evidence-based recommendations from the risk communication literature to promote understanding of probabilistic information

Recommendation
Avoid the use of verbal quantifiers
without numbers (eg, women who have
abnormal cells removed are slightly more
likely to have their baby early).

Avoid numerical ' 1 -in- $X$ ' formats to present the chance of an outcome (eg, 1 in 12 women will have an abnormal test result).

Avoid presenting estimates of risk
reduction in relative terms (eg, screening
cuts the risk of getting cervical cancer by
$75 \%)$.

Add simple graphical displays of numerical information (eg, icon arrays, where icons of different colours represent those affected and not affected by the risk).

\section{Rationale}

Interpretations of verbal quantifiers

vary across individuals and often

differ from interpretations intended by communicators. Verbal quantifiers can lead to overestimations of risks.

People tend to perceive the same probabilities as higher and more worrying when presented using ' 1 -in- $X$ ' ratios, as compared with numerically equivalent ' $\mathrm{N}$-in- $\mathrm{X}^{\star} \mathrm{N}$ ' ratios (eg, 10 in 120 women will have an abnormal test result) or percentages (eg, $8 \%$ of women will have an abnormal test result).

Relative risk differences can obscure the true magnitude of benefit or harm, as compared with absolute risk differences (eg, screening reduces the risk of getting cervical cancer from 20 in 1000 to 5 in 1000).

\section{Key references}

Budescu et $a^{77}$; Knapp et al ${ }^{5556}$; Peters et $a l^{57}$; Visschers et $a l^{53}$; Young and Oppenheimer ${ }^{58}$; Zipkin et a/ ${ }^{38}$

\section{Pighin et $a l^{7879}$; Sirota et $a /^{548081}$;} Trevena et al ${ }^{48}$; Zikmund-Fisher ${ }^{39}$

Akl et $\mathrm{al}^{82}$; Covey ${ }^{83}$; Fagerlin et $\mathrm{al}^{22}$; Gigerenzer et al ${ }^{21} 84$; Trevena et $a^{48}$; Zipkin et $a l^{38}$

Well-designed, simple graphs help to overcome difficulties in understanding numerical information and are often perceived as more appealing and easier to understand. 
personal autonomy and ensure that decisions are in line with invitees' personal values and circumstances-especially since healthy individuals can be adversely affected by screening. ${ }^{2526}$ Besides undermining the principle of autonomy, persuasive messages may have unintended negative effects, such as eliciting guilt and anxiety among invitees who decline the offer, anger among those who participate and are harmed as a result, and potential mistrust in communicators over time. ${ }^{202627}$

We investigated whether UK websites about cervical screening adhere to existing guidelines and policy recommendations concerning information content and appeals for informed decision-making (vs for participation), as well as to recommendations from the risk communication literature concerning quantitative presentation formats. Previous website analyses have primarily focused on breast cancer screening, and have generally assessed only website content ${ }^{25} 28$ or a specific aspect of presentation format (eg, consistency in the presentation of statistics on overdiagnosis ${ }^{29}$ ).

We used an established checklist of information items about cervical screening, ${ }^{30}$ building on earlier evaluations of breast cancer screening communications. ${ }^{25} 3132$ We assessed quantitative presentation formats considering the recommendations listed in table 1 . Additionally, we examined the type of appeals included in websites. In sum, our research questions were:

1. Do UK websites about cervical screening contain (a) key information about screening benefits, risks, possible results, and (b) cervical cancer statistics?

2. Do UK websites about cervical screening present probabilistic information using recommended formats?

3. Do UK websites about cervical screening contain appeals for participation and/or informed decision making?

\section{METHODS}

\section{Search strategy}

We used GoogleTrends to identify the most common Google search terms used in the UK to find information on cervical screening. We identified search terms related to 'cervical screening' in the 12 months prior to March 2017. We used 'cervical screening' based on the invitations from the UK's NHS. Related search terms included 'cervical cancer screening,' 'cervical smear' and 'smear test'. To determine which of these terms was more common, we used Wordtracker (https:/ /app.wordtracker. $\mathrm{com} /$ ), a commercial programme that estimates the relative frequency of Google search terms in a given period of time (see Ref. 33 for a similar procedure). The two most commonly used terms in the UK in the year before March 2017 were 'smear test' and 'cervical screening'.

\section{Website selection}

On 9 March 2017, we performed the Google search 'smear test' OR 'cervical screening' using the private Firefox browsing mode to prevent previously visited pages and cookies from influencing search results. Following Ghanouni $e t a l,{ }^{29}$ we examined the first five pages of results using the default of 10 results per page, (i.e., 50 links). This includes the vast majority of websites that users will access (see also Ref. 34). The full list of links is available at the Open Science Framework (https://doi. org/10.17605/OSF.IO/73GFN).

We excluded links that (1) targeted healthcare professionals and academics rather than laypeople (eg, technical reports, research articles), (2) reflected online media articles or press releases, (3) presented international information not applicable to the UK (eg, Wikipedia's overview of screening recommendations in different countries) and (4) were locally or regionally oriented. The latter links typically focused on basic practical aspects (eg, who is eligible for screening and logistics of making appointments for specific general practice surgeries), and often provided links for further information to national websites included in our analyses. We also excluded links that (5) were duplicate, (6) contained no or little information about cervical screening ( $<150$ words) and ( 7$)$ had no written materials (eg, YouTube video). Additionally, we included two websites identified through our knowledge of resources on cervical screening (Patient and Women's Health Concern). Although these websites did not appear in the Google search, they represent trusted UK resources that some women may access directly to learn about cervical screening. Figure 1 summarises the website selection process.

For the websites identified through Google, we coded the link listed in the search results. For the two additional websites, we coded the main link providing information about cervical screening. In all cases, we also coded sections on cervical screening within each website that could be directly accessed from the initial link, as well as other written materials directly accessible, including electronic leaflets, fact sheets and slideshows, which were all considered as part of the same resource in analyses. Websites were accessed between 20 March 2017 and 28 April 2017, except one website (BootsWebMD), which was accessed at a later date due to an oversight in the initial website selection. The date of accessing each website, corresponding links and estimated number of visits appears in supplementary materials (online supplementary table S1). Copies of PDF files reflecting the content of all websites at the time of access are available at the Open Science Framework.

\section{Coding of websites}

To code website content, we adapted a checklist of information items that was developed to analyse invitations for cervical screening. ${ }^{30}$ Following European and UK guidelines, ${ }^{18} 19$ the checklist included items about screening benefits, risks and possible results (eg, the possibility of an abnormal or an inadequate result). Following Kolthoff $e t a l^{30}$ the item on overdiagnosis/overtreatment included any reference to screening possibly detecting abnormal cells that may clear up on their own and/or leading to unnecessary treatment, regardless of 


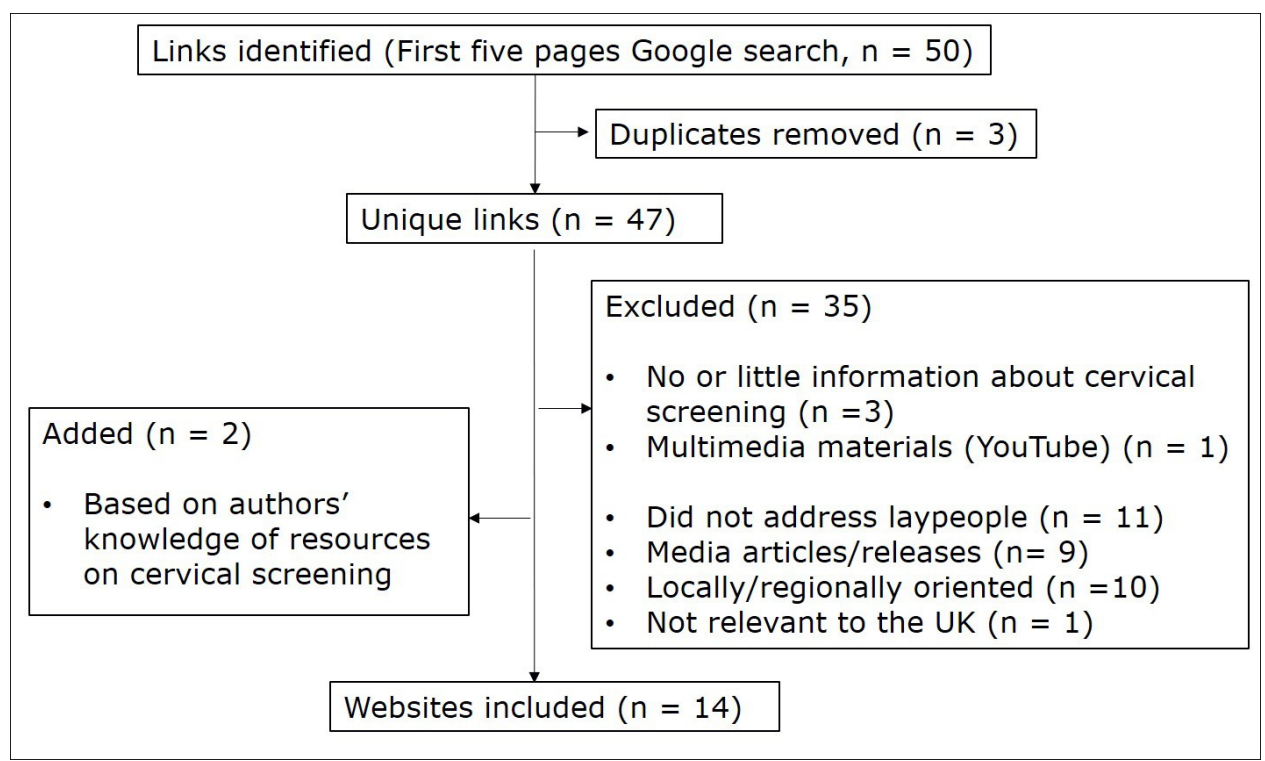

Figure 1 Flow chart representing the website selection process.

whether the treatment type was mentioned (see also Refs). ${ }^{3536}$ The checklist also included items about cervical cancer statistics (eg, lifetime risk of developing cervical cancer), which may be relevant for screening decisions. ${ }^{25} 3132$ After initial inspection of the websites, we added five items to the checklist to reflect commonly presented information that may also influence screening decisions (figure 2). Examples of all information items appear in supplementary materials (online supplementary table S2).
To assess quantitative presentation formats, we coded whether probabilistic information was presented using: no quantifiers, verbal quantifiers only, numerical quantifiers (1-in-X vs other numerical format) and/or graphs. We also coded whether any information about risk reduction was presented in relative terms. Multiple formats (eg, graphs accompanied by numbers) were present in some communication materials (online supplementary table S3, supplementary materials).

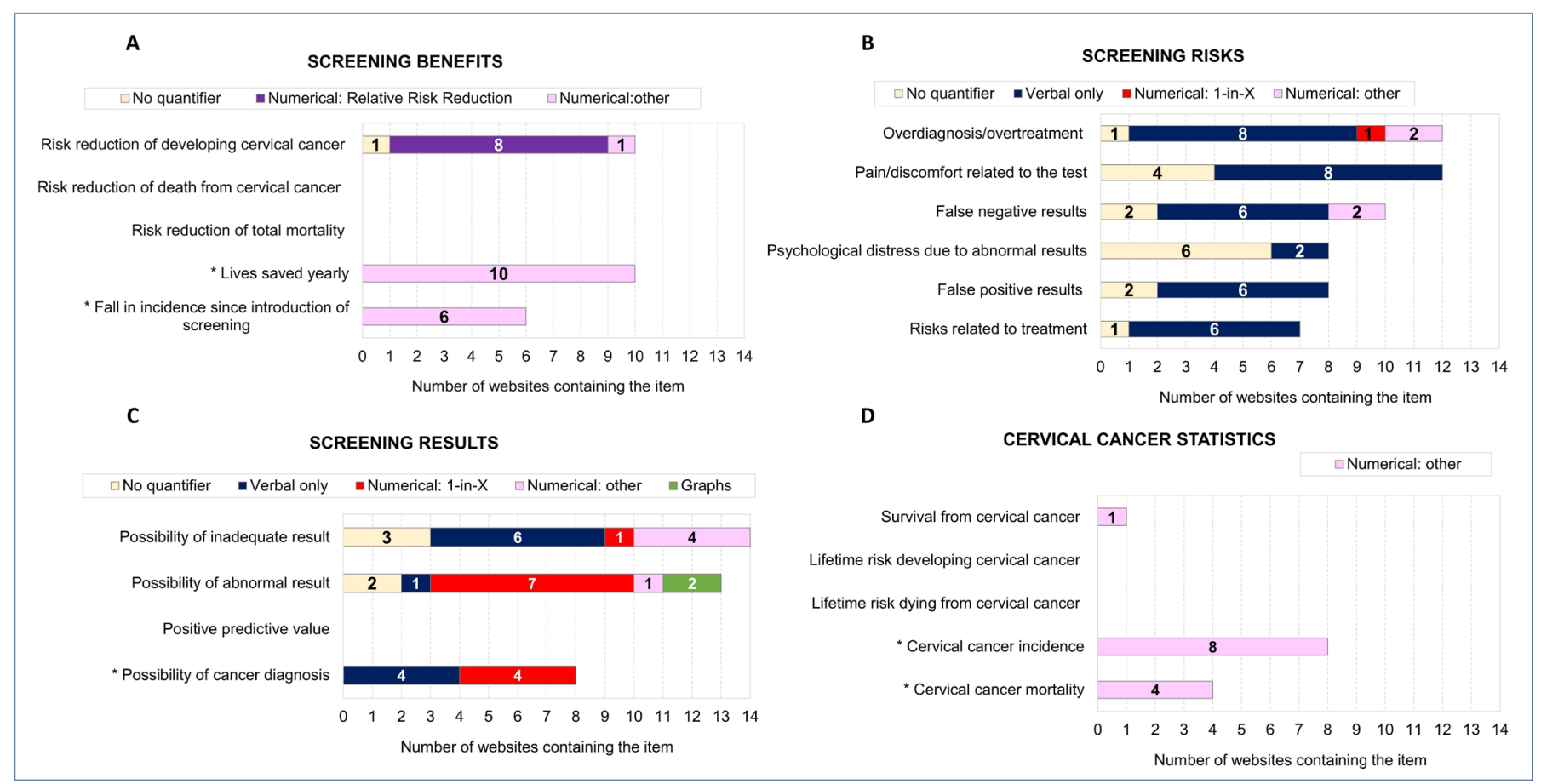

Figure 2 Results of analysis of content and quantitative presentation formats for information about (A) cervical screening benefits, (B) risks, (C) possible results and (D) cervical cancer statistics. A total of 14 websites were analysed. Items marked with an asterisk were added by the authors to the checklist by Kolthoff et $\mathrm{al} .{ }^{30}$ Details concerning instances in which multiple formats were used for a given information item appear in supplementary materials (online supplementary table S3). 
Finally, we coded appeals for informed decision-making (eg, 'deciding whether to have screening or not is your choice') and appeals for participation. Following Kolthoff et $a l^{30}$ the latter included direct encouragement for participation (eg, 'a smear test—have it') and statements presenting screening as necessary (eg, 'it's essential for women to have regular cervical screening tests') or important (eg, 'it is important to go for screening every time you are invited').

The first author (YO) read through all selected materials and applied the coding scheme. Three websites $(>20 \%)$ were coded by another author (SGS). Cohen's kappa was moderate to high on all codes except 'type of appeal' (0.18). Discrepancies for this code were caused by appeals for participation that were not prominent. These discrepancies were resolved through discussion, resulting in consensus. The average Cohen's kappa was 0.86 (range: $0.65-1$ ).

\section{Patient and public involvement}

Patients and/or public were not involved in the design or conduct of the study, which did not involve human participants.

\section{RESULTS}

\section{Website selection}

As described above, we identified 50 links through our Google search. After exclusions, 12 websites remained, including 4 websites from public health service providers in the UK's four countries (NHS Choices England, NHS Inform Scotland, Public Health Agency Northern Ireland, Public Health Wales), 1 private health service provider (Bupa UK), 3 cancer charities (Cancer Research UK, Jo's Cervical Cancer Trust, MacMillan Cancer Support), 2 additional charities (LGBT Foundation, Marie Stopes UK), 1 medical society (British Society for Colposcopy and Cervical Pathology) and 1 pharmacy (Boots WebMD). All websites from public health service providers included links to official invitation leaflets, which were coded with the corresponding website, as described above. As noted earlier, two additional websites (Patient and Women's Health Concern) were identified by the authors. Below, we present summary statistics across websites. Results for individual websites appear in supplementary materials (online supplementary table S3).

\section{Do UK websites about cervical screening contain (a) key information about screening benefits, risks, possible results and (b) cervical cancer statistics?}

We report on four main findings regarding website content. First, screening benefits were mentioned frequently, although the focus was primarily on risk reduction of developing cervical cancer (figure 2A). Estimates of risk reduction typically varied between $70 \%$ and $80 \%$. Based on Sasieni $e t a l,{ }^{37}$ two websites provided an estimated range of $60 \%-80 \%$, and another provided an estimate of $90 \%$ for the reduction in the risk of advanced cancer specifically. No websites explicitly mentioned risk reductions of death from cervical cancer or total mortality. Instead, benefits were often expressed in terms of the number of lives saved yearly (eg, 'cervical screening saves 5000 lives a year in the UK'; $\mathrm{n}=10$ websites), or in some cases referring to the fall in cervical cancer cases since the national screening programme was initiated (eg, 'since the screening programme was introduced in the 1980s, the number of cervical cancer cases has decreased by about $7 \%$ each year'; $n=6$ ).

Second, screening risks were also mentioned relatively frequently (figure 2B), but the specific risks and their descriptions varied across websites. The most commonly mentioned risks were overdiagnosis and overtreatment $(n=12)$, pain/discomfort related to the test $(n=12)$ and the possibility of false negatives $(n=10)$. Descriptions of overdiagnosis, however, generally focused on cell changes often clearing up and not progressing into cancer (eg, 'in most cases, the abnormal cells will disappear on their own'). The risk of unnecessary additional tests or treatment was only mentioned explicitly in seven cases, often in connection to justifications for current screening age ranges (eg, 'changes in a young woman's cervix are quite normal. In this situation, screening may lead to unnecessary treatment'). Most websites described the test as uncomfortable but not painful (eg, 'having the speculum put in may be a little uncomfortable, but it shouldn't hurt'), though one website did state that the test was potentially painful. Risks related to treatment were only mentioned in half of the websites, all of which referred to the possibility of premature birth. Two of them also noted the risk of stenosis (ie, the cervix becoming tightly closed after treatment).

Third, possible screening results were mentioned in most websites (figure 2C). Estimates of the likelihood of inadequate results varied between $1.7 \%$ and $3 \%$ and estimates of the likelihood of abnormal results ranged between $5 \%$ and $10 \%$. Additionally, the possibility of cancer diagnosis was mentioned in over half of the websites $(n=8)$ and was often reported as occurring in less than 1 in 1000 cases.

Finally, cervical cancer statistics such as lifetime risk of developing or dying from cervical cancer were not discussed, although some websites did provide details on cervical cancer incidence and mortality (figure 2D). Specific estimates of incidence varied across websites, and included yearly, daily, national and regional estimates (eg, 'each year in Northern Ireland, about 103 women are diagnosed with cervical cancer').

Do UK websites about cervical screening present probabilistic information using recommended formats?

Information was often not presented in recommended formats. First, the recommendation to avoid the use of verbal quantifiers without numbers ${ }^{38}$ was often not followed for information about risks (figure 2B). Risks related to treatment were quantified only verbally, generally using varying verbal quantifiers. For example, one 
website stated that 'women are slightly more likely to have their baby 1 to 2 months early', and another stated that 'women may have a higher risk of preterm delivery'. Other risks were also either only quantified verbally or not quantified at all, with the exception of overdiagnosis/overtreatment $(n=3)$ and false-negative results $(n=2)$.

Second, the recommendation to avoid ' 1 -in- $\mathrm{X}$ ' numerical formats ${ }^{39}$ was often violated for information about screening results. This was the most popular format for conveying the likelihood of abnormal results (figure 2C). Third, the recommendation to avoid presentations of relative risk reduction ${ }^{21}$ was violated in most cases in which an estimate of risk reduction was provided (figure 2A). Finally, the recommendation to add simple graphical displays ${ }^{40}$ was often not met. Only two websites contained graphs, each depicting the likelihood of abnormal results.

\section{Do UK websites about cervical screening contain appeals for participation and/or informed decision-making?}

Appeals for participation were present in most websites $(n=12)$. Specifically, appeals for participation without mention of informed decision-making occurred in half of the websites $(n=7)$. Several websites combined appeals for participation and informed decision-making $(\mathrm{n}=5)(\mathrm{eg}$, 'don't ignore your smear test, it could save your life' and 'taking part in cervical screening is your choice'). One website (NHS Choices England) referred to informed decision-making only ('deciding whether or not to have a cervical screening test is your choice'). The websites of the three remaining public health service providers included either both appeals for participation and informed decision-making (NHS Inform Scotland and Public Health Wales) or only appeals for participation (Public Health Agency Northern Ireland).

\section{DISCUSSION}

We investigated whether UK websites about cervical screening adhered to existing recommendations about content, quantitative presentation formats and type of appeals. We found that websites often followed recommendations from European and UK guidelines to include information about both benefits and risks of screening. ${ }^{17-19}$ However, specific content varied across websites. For example, only half of the websites explicitly referred to risks related to the treatment of abnormal cells. Additionally, probabilistic information was often presented in formats not recommended in the risk communication literature, including relative risk reductions to express screening benefits, and verbal quantifiers without numbers to express risks. Moreover, several websites encouraged screening participation without referring to informed decision-making, contrasting with current UK policy. ${ }^{141}$

Our finding that websites often included information about both benefits and risks of cervical screening contrasts with previous reports that breast screening websites lacked information about risks such as overdiagnosis. ${ }^{25} 28$ Recent emphasis in the UK on facilitating informed screening decisions ${ }^{14}{ }^{41}$ may have led to more comprehensive and balanced websites in recent years (see also Ref. 29). The presence of information about both screening benefits and risks is aligned with women's preferences, who often want to receive information on both aspects before their test. ${ }^{42}$ However, we also found that websites generally presented benefits and risks using different formats (numbers vs verbal quantifiers, respectively), potentially hindering benefit-risk comparisons. Additionally, our finding that specific content varied across websites suggests that women accessing different resources may come to different conclusions.

Our results also suggest that existing descriptions of benefits and risks may not always support understanding. For example, the concepts of overdiagnosis and overtreatment are often unfamiliar and counterintuitive to people ${ }^{43-45}$ highlighting the need to provide explanations that are accessible and transparent. Many websites did explain that cell changes often clear up on their own. This may help women to understand that cervical screening can lead to overdiagnosis of indolent abnormalities (potentially causing unnecessary anxiety or worries ${ }^{36}$ ), but not necessarily that it may result in unnecessary treatment. Moreover, the websites that explicitly mentioned overtreatment often did so in relation to women below the recommended screening age. Hence, those within the recommended age may incorrectly infer that such risk does not apply to them. We also found that many websites emphasised that screening 'saves lives'. It has been argued that such claims are misleading, and that communications should report reductions in cancer-specific mortality, overall mortality, as well as overall cancer deaths. ${ }^{46}$ Although there is evidence that cervical screening reduces cancer-specific mortality, ${ }^{3-7}$ determining its impact on overall mortality requires large trials with sufficient power to detect differences. ${ }^{47}$

Our finding that probabilistic information was often presented in non-recommended formats is concerning as this may cause misperceptions, even among educated audiences. The use of relative risk reductions to express screening benefits can hinder understanding and increase risk perceptions, relative to presentations of absolute risk reduction. ${ }^{38} 48$ Although information about baseline risk (ie, the absolute risk without screening) could reduce the biassing effect of relative risks ${ }^{49}$ (but see Ref. 50), such beneficial impact may be limited to baseline risks presented in frequency formats (vs probabilities) ${ }^{51} 52$ and to more (vs less) numerate recipients. ${ }^{52}$ Moreover, we did not find such information in any of the websites. It has been argued, however, that presentations of relative risks may be considered for low-probability risks with high impact (eg, an earthquake), to prevent people from neglecting such risks altogether. ${ }^{2453}$

Additionally, our results suggest that women may overestimate the likelihood of abnormal screening results due to the use of ' 1 -in- $X$ ' formats ( $\mathrm{eg}$, ' 1 in 12 women will have an abnormal test result') ${ }^{54}$ Similarly, screening risks 
expressed using verbal quantifiers without numbers may be overestimated.$^{55-58}$ The absence of numerical information can also lead people to perceive communications as less credible and trustworthy. ${ }^{53} 5960$ Although some risks are hard to quantify (eg, psychological distress), estimates are available for risks such as preterm birth or overtreatment, from observational studies and meta-analyses. ${ }^{91113}$ The best available evidence could be presented in transparent fact boxes-a tabular format that facilitates comparisons of outcomes in groups of screened versus unscreened individuals. ${ }^{61}$ Fact boxes can also include simple graphs such as icon arrays to allow visual comparison of quantities, ${ }^{62}$ which could be especially beneficial for people with low numeracy. Yet, graphs were seldom used in cervical screening websites.

Finally, our finding that some websites encouraged screening without mentioning informed decision-making contrasts with current UK policy, which emphasises that communications can recommend screening, but should acknowledge that not accepting the offer is a reasonable choice. ${ }^{1420}$ Although there is largely agreement that benefits of cervical screening outweigh potential risks, ${ }^{51415}$ persuasive messages raise ethical concerns to the extent that harms are possible. ${ }^{18} 2663$ Persuasive messages may also contribute to widespread enthusiasm for cancer screening, ${ }^{64} 65$ discouraging people from reasoning about their screening choices. ${ }^{26}$ Moreover, while guidelines for screening communications tend to focus on health service providers, ${ }^{17-19}$ conflicting recommendations from different sources might create public confusion, negative beliefs about recommendations and scientific research, and potentially reduce screening intentions. ${ }^{667}$

Strengths of our analyses include their comprehensiveness and systematic examination of website content using an established checklist of information items, ${ }^{30}$ and recommendations from the literature for quantitative presentation formats. Additionally, our analyses included most of the websites that UK citizens may realistically access, as our strategy for website selection was based on the top results identified by the most commonly used Google search terms. Our approach could be used by researchers and practitioners to evaluate the content and format of websites about related topics (eg, other types of screening) in different countries. This would allow comparisons of websites for countries that have organised versus opportunistic screening. For example, website content may be more heterogeneous in countries such as the USA, which do not have organised screening. It would also be interesting to compare different platforms (eg, desktop websites vs mobile tailored versions), and to examine whether different design features (eg, information position ${ }^{68}$ ) affect information readability, saliency or users' information seeking behaviour .

Limitations of our study include that our analyses may not have covered all information that is potentially relevant for screening decisions. For example, we did not assess whether websites discussed HPV, or the uncertainty associated with estimated benefits and risks.
Communicating uncertainty can be challenging ${ }^{69-71}$ but is important for informed decision-making. ${ }^{16} 1741$ Conversely, it is possible that not all codes assessed are essential for informed screening decisions. We also did not directly examine whether users can make informed screening decisions based on the different websites. It is likely, however, that following guidelines and recommendations from the literature will facilitate better understanding and more informed decisions. Explicit appeals to informed decision-making may also encourage users to evaluate the content, but of course do not guarantee that informed decisions will occur.

Future work should aim to identify essential information items for informed decisions about screening, considering both experts' and women's views. As noted by Ghanouni et $a l^{72}$ guidelines often do not provide detailed advice on which specific screening benefits, risks, or results should be discussed in communications. Moreover, experts may not fully agree on the relative importance of different information items, and women may have different information needs and preferences, including about whether and when to receive information about further tests. Providing too much unfamiliar information initially may also overwhelm screening invitees, and potentially distract them from key information necessary for decision-making. ${ }^{22} 73$ More research is also needed to identify how to best convey the individual significance of the population-level estimates included in screening communications, which may be hard for people to understand. ${ }^{74}$ Finally, future studies should also examine how screening decisions (eg, intentions to participate ${ }^{75}$ ) are affected by information about specific benefits and/or risks presented using different formats, building on the risk communication literature $\left(\mathrm{eg},{ }^{76}\right)$. Such work would provide valuable insights to inform the design of evidence-based public communications about screening.

\section{Twitter Yasmina Okan @yasminaokan}

Acknowledgements The authors are grateful to Mirta Galesic and Erika Waters for their valuable comments and suggestions. They would also like to thank the reviewers (Jo Waller, Felix G. Rebitschek, and Seok Won Jin) for their constructive feedback.

Contributors YO conceived the research, acquired and analysed the data, and drafted the initial version of the manuscript. SGS assisted with data analyses and interpretation. WBdB contributed to data interpretation. All authors contributed to study design, revised the manuscript critically and approved the final version.

Funding This work was supported by a Population Research Fellowship awarded by Cancer Research UK to Yasmina Okan (reference C57775/A22182). SS is supported by Yorkshire Cancer Research. WBdB was partially supported by a grant from the Swedish Riksbanken Jubilieumsfond programme on Science and Proven Experience.

Disclaimer The funding agencies had no involvement in designing the study, data collection, analysis and interpretation, writing the report, or the decision to submit the article for publication.

Competing interests SGS is an academic consultant for Luto, who are not involved in any of the websites reviewed in this manuscript.

Patient consent for publication Not required.

Provenance and peer review Not commissioned; externally peer reviewed. 
Data availability statement Data are available in a public, open access repository.

Open access This is an open access article distributed in accordance with the Creative Commons Attribution 4.0 Unported (CC BY 4.0) license, which permits others to copy, redistribute, remix, transform and build upon this work for any purpose, provided the original work is properly cited, a link to the licence is given, and indication of whether changes were made. See: https://creativecommons.org/ licenses/by/4.0/.

\section{ORCID iDs}

Yasmina Okan http://orcid.org/0000-0001-7963-1363

Wändi Bruine de Bruin http://orcid.org/0000-0002-1601-789X

\section{REFERENCES}

1 Crosbie EJ, Einstein MH, Franceschi S, et al. Human papillomavirus and cervical cancer. The Lancet 2013;382:889-99.

2 Muñoz N, Bosch FX, de Sanjosé S, et al. Epidemiologic classification of human papillomavirus types associated with cervical cancer. $N$ Engl J Med 2003;348:518-27.

3 Peirson L, Fitzpatrick-Lewis D, Ciliska D, et al. Screening for cervical cancer: a systematic review and meta-analysis. Syst Rev 2013;2:35.

4 Nygård M. Screening for cervical cancer: when theory meets reality. BMC Cancer 2011;11:240.

5 Esserman LJ, Thompson IM, Reid B. Overdiagnosis and overtreatment in cancer: an opportunity for improvement. JAMA 2013;310:797-8

6 Peto J, Gilham C, Fletcher O, et al. The cervical cancer epidemic that screening has prevented in the UK. The Lancet 2004;364:249-56.

7 Landy R, Pesola F, Castañón A, et al. Impact of cervical screening on cervical cancer mortality: estimation using stage-specific results from a nested case-control study. Br J Cancer 2016;115:1140-6.

8 Smittenaar CR, Petersen KA, Stewart K, et al. Cancer incidence and mortality projections in the UK until 2035. Br J Cancer 2016;115:1147-55.

9 Tainio K, Athanasiou A, Tikkinen KAO, et al. Clinical course of untreated cervical intraepithelial neoplasia grade 2 under active surveillance: systematic review and meta-analysis. BMJ 2018;360.

10 McCredie MRE, Sharples KJ, Paul C, et al. Natural history of cervical neoplasia and risk of invasive cancer in women with cervical intraepithelial neoplasia 3: a retrospective cohort study. Lancet Oncol 2008:9:425-34

11 Castanon A, Landy R, Brocklehurst P, et al. Risk of preterm delivery with increasing depth of excision for cervical intraepithelial neoplasia in England: nested case-control study. BMJ 2014;349:g6223.

12 Sasieni $P$, Castanon A, Landy R, et al. Risk of preterm birth following surgical treatment for cervical disease: Executive summary of a recent symposium. BJOG 2016;123:1426-9.

13 Kyrgiou M, Athanasiou A, Kalliala IEJ, et al. Obstetric outcomes after conservative treatment for cervical intraepithelial lesions and early invasive disease. Cochrane Database Syst Rev 2017;11.

14 Forbes LJL, Ramirez AJ. Communicating the benefits and harms of cancer screening. Curr Oncol Rep 2014;16:382.

15 Moyer VA, U.S. Preventive Services Task Force. Screening for cervical cancer: U.S. preventive services Task force recommendation statement. Ann Intern Med 2012;156:880-91.

16 Hersch J, Nickel B, Ghanouni A, et al. Improving communication about cancer screening: moving towards informed decision making. Public Health Res Pract 2017;27:e2731728.

17 UK National Screening Committee. Guidance for the development, production and review of information to support UK population screening programmes, 2018. Available: https://www.gov.uk/ government/publications/uk-national-screening-committeeinformation-development-guidance [Accessed 17 Aug 2018]

18 European Commission. European guidelines for quality assurance in cervical cancer screening. 2nd edition. Luxembourg: Office for Official Publications of the European Communities, 2008. http:// screening.iarc.fr/doc/ND7007117ENC_002.pdf

19 General Medical Council. Consent: patients and doctors making decisions together, 2008. Available: https://www.gmc-uk.org/ethicalguidance/ethical-guidance-for-doctors/consent [Accessed 17 Aug 2018].

20 Entwistle VA, Carter SM, Trevena L, et al. Communicating about screening. BMJ 2008;337:a1591-91.

21 Gigerenzer G, Gaissmaier W, Kurz-Milcke E, et al. Helping doctors and patients make sense of health statistics. Psychol Sci Public Interest 2007;8:53-96.

22 Fagerlin A, Zikmund-Fisher BJ, Ubel PA. Helping patients decide: ten steps to better risk communication. J Natl Cancer Inst 2011;103:1436-43.
23 Schwartz LM, Woloshin S, Black WC, et al. The role of numeracy in understanding the benefit of screening mammography. Ann Intern Med 1997;127:966-72

24 Spiegelhalter D. Risk and uncertainty communication. Annu Rev Stat Appl 2017;4:31-60.

25 Jørgensen KJ, Gøtzsche PC. Presentation on websites of possible benefits and harms from screening for breast cancer: cross sectional study. BMJ 2004;328.

26 Rychetnik L, Carter SM, Abelson J, et al. Enhancing citizen engagement in cancer screening through deliberative democracy. $J$ Natl Cancer Inst 2013;105:380-6.

27 Raffle AE. Information about screening - is it to achieve high uptake or to ensure informed choice? Health Expect 2001;4:92-8.

28 Attena F, Cancellieri M, Pelullo CP. Scarce information about breast cancer screening: an Italian websites analysis. Medicine 2016;95:e5615

29 Ghanouni A, Meisel SF, Hersch J, et al. Information on 'Overdiagnosis' in Breast Cancer Screening on Prominent United Kingdom- and Australia-Oriented Health Websites. PLoS One 2016;11:e0152279.

30 Kolthoff SK, Hestbech MS, Jørgensen KJ, et al. Do invitations for cervical screening provide sufficient information to enable informed choice? A cross-sectional study of invitations for publicly funded cervical screening. J R Soc Med 2016;109:274-81.

31 Slaytor EK, Ward JE. How risks of breast cancer and benefits of screening are Communicated to women: analysis of 58 pamphlets. BMJ 1998;317:263-4.

32 Jørgensen KJ, Gøtzsche PC. Content of invitations for publicly funded screening mammography. BMJ 2006;332:538-41.

33 McHugh SM, Corrigan M, Morney N, et al. A quantitative assessment of changing trends in Internet usage for cancer information. World $\mathrm{J}$ Surg 2011;35:253-7.

34 Liebl P, Seilacher E, Koester M-J, et al. What cancer patients find in the internet: the visibility of evidence-based patient information - analysis of information on German websites. Oncol Res Treat 2015;38:212-8.

35 Phillips K, Hersch J, Turner R, et al. The influence of the 'cancer effect' on young women's responses to overdiagnosis in cervical screening. Patient Educ Couns 2016;99:1568-75.

36 Brodersen J, Schwartz LM, Heneghan C, et al. Overdiagnosis: what it is and what it isn't. Bmi Ebm 2018;23:1-3.

37 Sasieni P, Castanon A, Cuzick J, et al. Effectiveness of cervical screening with age: population based case-control study of prospectively recorded data. BMJ 2009;339:b2968.

38 Zipkin DA, Umscheid CA, Keating NL, et al. Evidence-Based risk communication: a systematic review. Ann Intern Med 2014;161:270-80.

39 Zikmund-Fisher BJ. Continued use of 1 -in-X risk communications is a systemic problem. Med Decis Making 2014;34:412-3.

40 Garcia-Retamero R, Cokely ET. Designing visual aids that promote risk literacy: a systematic review of health research and evidencebased design heuristics. Hum Factors 2017;59:582-627.

41 Forbes LJL, Ramirez A-J, Expert group on Information about Breast Screening. Offering informed choice about breast screening. J Med Screen 2014;21:194-200.

42 Dieng M, Trevena L, Turner RM, et al. What Australian women want and when they want it: cervical screening testing preferences, decision-making styles and information needs. Health Expect 2013;16:177-88.

43 Waller J, Whitaker KL, Winstanley K, et al. A survey study of women's responses to information about overdiagnosis in breast cancer screening in Britain. Br J Cancer 2014;111:1831-5.

44 Nagler RH, Franklin Fowler E, Gollust SE. Women's awareness of and responses to messages about breast cancer overdiagnosis and overtreatment. Med Care 2017;55:879-85.

45 Ghanouni A, Renzi C, Waller J. Improving public understanding of 'overdiagnosis' in England: a population survey assessing familiarity with possible terms for labelling the concept and perceptions of appropriate terminology. BMJ Open 2018;8:e021260.

46 Gigerenzer $\mathrm{G}$. Full disclosure about cancer screening. BMJ 2016;352.

47 Prasad V, Lenzer J, Newman DH. Why cancer screening has never been shown to "save lives" - and what we can do about it. BMJ 2016;352.

48 Trevena LJ, Zikmund-Fisher BJ, Edwards A, et al. Presenting quantitative information about decision outcomes: a risk communication primer for patient decision aid developers. BMC Med Inform Decis Mak 2013;13 Suppl 2:S7.

49 Natter HM, Berry DC. Effects of presenting the baseline risk when communicating absolute and relative risk reductions. Psychol Health Med 2005;10:326-34. 
50 Sorensen L, Gyrd-Hansen D, Kristiansen IS, et al. Laypersons' understanding of relative risk reductions: randomised cross-sectional study. BMC Med Inform Decis Mak 2008;8:31.

51 Covey J. The effects of absolute risks, relative risks, frequencies, and probabilities on decision quality. J Health Commun 2011;16:788-801.

52 Bodemer N, Meder B, Gigerenzer G. Communicating relative risk changes with baseline risk: presentation format and numeracy matter. Med Decis Making 2014;34:615-26.

53 Visschers VHM, Meertens RM, Passchier WWF, et al. Probability information in risk communication: a review of the research literature. Risk Anal 2009;29:267-87.

54 Sirota M, Juanchich M, Bonnefon J-F. "1-in-X" bias: "1-in-X" format causes overestimation of health-related risks. J Exp Psychol Appl 2018;24:431-9.

55 Knapp P, Raynor DK, Berry DC. Comparison of two methods of presenting risk information to patients about the side effects of medicines. Qual Saf Health Care 2004;13:176-80.

56 Knapp P, Gardner PH, Woolf E. Combined verbal and numerical expressions increase perceived risk of medicine side-effects: a randomized controlled trial of EMA recommendations. Health Expect 2016;19:264-74.

57 Peters E, Hart PS, Tusler M, et al. Numbers matter to informed patient choices: a randomized design across age and numeracy levels. Med Decis Making 2014;34:430-42.

58 Young S, Oppenheimer DM. Effect of communication strategy on personal risk perception and treatment adherence intentions. Psychol Health Med 2009;14:430-42.

59 Jenkins SC, Harris AJL, Lark RM. When unlikely outcomes occur: the role of communication format in maintaining communicator credibility. J Risk Res 2019;22:537-54.

60 Gurmankin AD, Baron J, Armstrong K. The effect of numerical statements of risk on trust and comfort with hypothetical physician risk communication. Med Decis Making 2004;24:265-71.

61 McDowell M, Rebitschek FG, Gigerenzer G, et al. A simple tool for communicating the benefits and harms of health interventions: a guide for creating a fact box. MDM Policy Pract 2016;1:1-10.

62 McDowell M, Gigerenzer G, Wegwarth O, et al. Effect of tabular and icon fact box formats on comprehension of benefits and harms of prostate cancer screening: a randomized trial. Med Decis Making 2019;39:41-56.

63 Woloshin S, Schwartz LM, Black WC, et al. Cancer screening campaigns--getting past uninformative persuasion. N Engl J Med 2012;367:1677-9.

64 Schwartz LM, Woloshin S, Fowler FJ, et al. Enthusiasm for cancer screening in the United States. JAMA 2004;291:71-8.

65 Waller J, Osborne K, Wardle J. Enthusiasm for cancer screening in Great Britain: a general population survey. Br J Cancer 2015;112:562-6.

66 Nagler RH. Adverse outcomes associated with media exposure to contradictory nutrition messages. $J$ Health Commun 2014;19:24-40.

67 Han PKJ, Kobrin SC, Klein WM, et al. Perceived ambiguity about screening mammography recommendations: association with future mammography uptake and perceptions. Cancer Epidemiology Biomarkers \& Prevention 2007;16:458-66.

68 Cheng C, Dunn M. How well are health information websites displayed on mobile phones? implications for the readability of health information. Health Promot J Austr 2017;28:15-20.
69 Longman T, Turner RM, King M, et al. The effects of communicating uncertainty in quantitative health risk estimates. Patient Educ Couns 2012;89:252-9.

70 Sladakovic J, Jansen J, Hersch J, et al. The differential effects of presenting uncertainty around benefits and harms on treatment decision making. Patient Educ Couns 2016;99:974-80.

71 Han PKJ. Conceptual, methodological, and ethical problems in communicating uncertainty in clinical evidence. Med Care Res Rev 2013;70(1 Suppl):14S-36.

72 Ghanouni A, Renzi C, Meisel SF, et al. Common methods of measuring 'informed choice' in screening participation: Challenges and future directions. Prev Med Rep 2016;4:601-7.

73 Peters E, Dieckmann N, Dixon A, et al. Less is more in presenting quality information to consumers. Med Care Res Rev 2007:64:169-90.

74 Waller J, Douglas E, Whitaker KL, et al. Women's responses to information about overdiagnosis in the UK breast cancer screening programme: a qualitative study. BMJ Open 2013;3:e002703.

75 Hestbech MS, Gyrd-Hansen D, Kragstrup J, et al. Effects of numerical information on intention to participate in cervical screening among women offered HPV vaccination: a randomised study. Scand $J$ Prim Health Care 2016;34:401-19.

76 Bunge M, Mühlhauser I, Steckelberg A. What constitutes evidencebased patient information? overview of discussed criteria. Patient Educ Couns 2010;78:316-28.

77 Budescu DV, Por H-H, Broomell SB, et al. The interpretation of IPCC probabilistic statements around the world. Nat Clim Chang 2014;4:508-12.

78 Pighin S, Savadori L, Barilli E, et al. The 1-in-X effect on the subjective assessment of medical probabilities. Med Decis Making 2011;31:721-9.

79 Pighin S, Savadori L, Barilli E, et al. Communicating Down syndrome risk according to maternal age: "1-in- $X$ " effect on perceived risk. Prenat Diagn 2015;35:777-82.

80 Sirota M, Juanchich M, Kostopoulou O, et al. Decisive evidence on a smaller-than-you-think phenomenon: revisiting the " 1 -in-X" effect on subjective medical probabilities. Med Decis Making 2014;34:419-29.

81 Sirota M, Juanchich M, Petrova D, et al. Health Professionals Prefer to Communicate Risk-Related Numerical Information Using "1-in-X" Ratios. Med Decis Making 2018;38:366-76.

82 Akl EA, Oxman AD, Herrin J, et al. Using alternative statistical formats for presenting risks and risk reductions. Cochrane Database Syst Rev 2011;58.

83 Covey J. A meta-analysis of the effects of presenting treatment benefits in different formats. Med Decis Making 2007;27:638-54

84 Gigerenzer G, Edwards A. Simple tools for understanding risks: from innumeracy to insight. BMJ 2003;327:741-4.

85 Galesic M, Garcia-Retamero R, Gigerenzer G. Using icon arrays to communicate medical risks: overcoming low numeracy. Health Psychol 2009;28:210-6.

86 Okan Y, Garcia-Retamero R, Cokely ET, et al. Individual differences in graph literacy: overcoming denominator neglect in risk comprehension. J Behav Decis Mak 2012;25:390-401.

87 Paling J. Strategies to help patients understand risks. BMJ 2003;327:745-8.

88 Schapira MM, Nattinger AB, McHorney CA. Frequency or probability? A qualitative study of risk communication formats used in health care. Med Decis Making 2001;21:459-67. 